\section{Chirurgie der Gallenwege}

\section{J.-F. Gigot}

München, Urban \& Fischer bei Elsevier, 2007,160 S., 246 farb. Abb., 79,95 EUR

ISBN 978-3-437-24300-4

Das 2005 in Französisch erschienene Buch des Autors hat offenbar so viel Resonanz gefunden, dass einer der größten internationale Verlage sehr bald seine Übersetzung in Deutsch veranlasst hat. Schon im Vorwort gerät Henri Bismuth, einer der bekanntesten hepatobiliären Chirurgen, in eine sehr lobende und fast schwärmerische Beschreibung über seine Ausstattung und seinen Inhalt. Auch der kritische Leser ist nach der Lektüre des Werks begeistert. Betrachtet man nur den chirurgisch signifikanten Teil der Gallenwege, nämlich den extrahepatischen Anteil, so handelt sich nur um einen sehr kleinen Teil des Verdauungsapparats: ein feiner Kanal von zirka $10 \mathrm{~cm}$ Länge mit einer Aussackung, der Gallenblase. Auch die Pathologie ist begrenzt: Steine und Krebs (wenn man die seltene zystische Degeneration des Choledochus nicht berücksichtigt). Und dennoch wurden über diesen kleinen Bereich und für die zwei Erkrankungen seit mehr als 100 Jahren so viele Eingriffe beschrieben und Operationstechniken kontrovers diskutiert. Was wurden nicht alles für Neuerungen beschrieben, z.B. die berüchtigte Odditis, die die chirurgische Behandlung der biliären Lithiasis in den 1950er bis 1970er Jahren dominiert hat und mittlerweile verschwunden ist. Vor weit mehr als einem Jahrzehnt kam es dennoch durch das laparokopische Vorgehen zu einer chirurgischen Revolution, die alles Bisherige völlig verändert hat, obwohl die diagnostische Laparoskopie mit Biopsie und Gallenblasenpunktion mehr als 50 Jahre vorher bereits von Kalk beschrieben und angewandt worden war und so lange vor den Augen der konventionellen Chirurgen weiter schlummerte. In diesem richtigen Moment, in dem wieder Ruhe herrscht, hat der Verfasser ein Werk über die aktuellen Techniken der biliären Chirurgie von der Cholezystektomie bis zur Chirurgie der Gallentumoren herausgegeben. Dieser erfahrene Autor und hepatobiliäre Chirurg hat genügend Expertise in der konventionellen Chirurgie und beherrscht daher auch das laparoskopische Vorgehen. Alle wichtigen Techniken sind in sehr detaillierter und übersichtlicher Form didaktisch überzeugend und gekonnt illustriert beschrieben. Das Buch vermittelt darüber hinaus einen universellen Geist, da es durch seine Kenntnis dessen, was in anderen Ländern als traditionelle Chirurgenschule gilt, die weltweit angewand- ten Alternativen mit einbezieht. Dabei wird er von wenigen chirurgischen Kollegen aus seiner direkten Umgebung und dem französischen Sprachraum unterstützt; letztere Chirurgen fördern dabei sogar junge Talente. Das Werk wird daher ohne Einschränkung sehr empfohlen, und sein Preis ist günstig.

Karl-Joseph Paquet, Bad Kissingen

\section{Plastische und Rekonstruktive Chirurgie der Brust}

\section{C.J. Gabka, H. Bohmert}

Stuttgart, Thieme, 2006, 2., aktualis. Aufl. IX + 281 S., 694 farb. Abb, 3 Tab., 179,95 EUR

ISBN 978-3-13-100562-5

Das vor 11 Jahren herausgegebene Lehrbuch mit Farbatlas und Operationslehre für Brustchirurgie wurde sehr gut angenommen, zumal sich nirgendwo eine gleichermaßen photographisch und zeichnerisch gelungenere Darstellung der ausführlich beschriebenen Operationstechniken der Brustrekonstruktion findet. Dies wird auch dadurch bestätigt, dass 2 Jahre später eine englische Ausgabe erschienen ist und eine ebensolche in Spanisch in Vorbereitung ist. Inzwischen wurden rasant neue Techniken entwickelt und die Notwendigkeit plastischer Brustchirurgie bei onkologischen Operationen besonders durch die Entwicklung Organ erhaltender und rekonstruktiver Operationen bei der onkologischen Brustchirurgie immer evidenter. Eine Neuauflage bzw. ausgiebige Bearbeitung dieses Werks war daher erforderlich und erfolgte durch den Bohmert-Schüler Gabka und den Autor selbst. Inhaltlich beginnt sie mit der Anatomie der Brust und der Darstellung der Brustimplantate und setzt sich fort mit der Augmentations- und Reduktionsplastik. Es folgt die Besprechung und Darstellung der rekonstruktiven Chirurgie der Brust mit der Beschreibung von Deformitäten, der sekundären Brustrekonstruktion durch Implantate, Musculus-latissimus-dorsi-, TRAM/DIEP-, S-GAP- und TMG(tranversaler myokutaner Gracilis)-Lappen sowie der bilateralen Brust- und MamillenAreola-Rekonstruktion. In einem ausführlichen Kapitel wird die onkologische Chirurgie der Brust mit der prophylaktischen subkutanen Mastektomie, der modifiziert radikalen Mastektomie, der Brust erhaltenden Behandlung und der Sofortrekonstruktion der Brust mit Implantaten, Haut- und Haut-Muskel-Lappen abgehandelt. Letzteres Kapitel hat heute eine besonders große Bedeutung. Das Werk gibt jungen 
plastischen Chirurgen bzw. Operateuren eine praktische Anleitung für die Operationsmethoden, die sich als praktikabel, Erfolg versprechend und patientenorientiert erwiesen haben. Wenn es erforderlich ist, erfolgt eine kritische Auseinandersetzung bezüglich Indikation, Strategie und Technologien. Das Buch wäre ohne die Mitarbeit des Meisters der Perforator-Lappen-Brustrekonstruktion P. Blondeel und der «Fans» des differenzierten Einsatzes des TMG-Lappens, Schoeller und Wechselberger, nicht so perfekt und lesenswert geworden. Es ist nicht nur inhaltlich und didaktisch, sondern auch in seiner Illustration ziemlich einmalig und wird seinen Erfolgsweg nicht nur im deutschsprachigen Raum, sondern ebenso bei seiner zu erwartenden Übersetzung in Englisch und Spanisch auf der ganzen Welt weiter fortsetzen. Sein Preis ist in Anbetracht seiner großzügigen und besonders guten Ausstattung angemessen. Eine besondere Empfehlung hieße, Eulen nach Athen zu tragen.

Karl-Joseph Paquet, Bad Kissingen

\section{Wundatlas. Kompendium der komplexen Wundbehandlung}

\section{H. Lippert (Hrsg.)}

Stuttgart, Thieme, 2006, 2., überarb. Aufl. XII + 252 S.,

554 Abb., 99,95 EUR

ISBN 978-3-13-140832-7

Die Wunde ist stets Anlass zur menschlichen Hilfeleistung und steht daher im Mittelpunkt der ärztlichen Tätigkeit. Trotz einer scheinbaren Routine und Statik bei der Wundbehandlung sind die erzielten Fortschritte auf diesem Gebiet gewaltig. Deswegen war eine vollständige Überarbeitung bzw. Neuauflage des Buchs notwendig. Dabei darf die Vereinfachung der Wundbehandlung in den letzten Jahren keineswegs dazu verleiten, die erforderliche Sorgfalt und Differenzierung der Therapie zu vernachlässigen. Wunden infolge von Naturkatastrophen, Explosionen und Terrorakten, deren Häufigkeit in den letzten Jahrzehnten spürbar zugenommen hat und deren Behandlung zunehmend an Bedeutung gewinnt, werden daher in diesem Atlas erstmalig berücksichtigt. Dies gilt auch für Besonderheiten bei Gesichts-, Augen-, Schuss- und Explosionsverletzungen, bei Wunden im Perinealbereich sowie bei der Wundbehandlung der nicht selten betroffenen Kinder. Darüber hinaus wird auf forensische Aspekte der Wundbeurteilung eingegangen; ebenso wird die Krankenpflege und Qualitätssicherung in einem gesonderten Kapitel besprochen. Die Behandlung chronischer und daher besonders kostenträchtiger Wunden, die interdisziplinäre Diagnostik und Therapie des diabetischen Fußsyndroms und die Wundheilungskonzepte im Rahmen der Angioplastie und BypassChirurgie werden ebenso angesprochen. Berücksichtigt wird auch die Vakuumbehandlung, mit der erhebliche Fortschritte erreicht wurden, die Weiterentwicklung von Wundauflagen, die Prophylaxe und Therapie von Narben und neue Entwicklungen wie autologe, epitheliale Suspensionen und azelluläre Gewebsersatzprodukte bei der Behandlung von Verbrennungswunden. Die Wundbehandlung ist für alle medizinischen Fachrichtungen von großer Bedeutung. Dies zwingt zu interdisziplinären Therapiekonzepten und zur engen ärztlichpflegerischen Kooperation. Mit einem scharfen Blick auf diese hohen Ansprüche ist dem Autor mit dieser Neuauflage, die einen hervorragenden Überblick über alle modernen Methoden dieses Gebiets bietet, ein großer Wurf gelungen. Die vorgetragenen rationalen und konsequenten Strategien zur Diagnostik und Therapie überzeugen. Dabei ist die Gliederung didaktisch geschickt, übersichtlich und klar. Sie wird durch zahlreiche Farbabbildungen unterstützt, die die gebotene Information verdeutlichen und ergänzen. Dieses Werk wird daher nicht nur vom Rezensenten, sondern auch von der Deutschen Gesellschaft für Wundheilung und Wundbehandlung e. V. sehr empfohlen. Sein Preis ist in Anbetracht der großzügigen und umfangreichen Ausstattung sehr günstig.

Karl-Joseph Paquet, Bad Kissingen

\section{Lehratlas der Proktologie. Diagnostik, Therapie, Fallbeispiele}

\section{H. Rohde}

Stuttgart, Thieme, 2006, VIII + 237 S., 705 Farbphotos, 99,95 EUR

ISBN 978-3-13-140881-5

Auf dem Büchermarkt gibt es zwar einige Lehrbücher über Proktologie; mit diesem Atlas wird jedoch etwas Neues und Besonderes geboten. Schon seine Entstehung ist einmalig und doch konsequent: Ausgangspunkt waren Beispiele des Autors für die Rubrik MEDIQUIZ in der Deutschen Medizinischen Wochenschrift und die Reaktionen der Leser auf diese Beispiele, die nicht nur lobende Zustimmung, sondern auch wütende Proteste und gehässige Anmerkungen beinhalteten. Natürlich war dies eine unvergessliche Anregung für den Autor, der stets die Erstbeobachtungen und Verläufe seiner Patienten mit Photos dokumentiert hatte, dem aufgeschlossenen und kritischen Medizinpublikum seine umfangreichen Erfahrungen umfassend mitzuteilen. Dieser Schatz bildete die Grundlage für dieses Werk, das den Leser in die richtige proktologische Untersuchung, den normalen und krankhaften Befund und die praxisnahe Erhebung und Dokumentation des Letzteren einführt und bis ins Detail darstellt. Dabei weist er immer wieder auf den Mangel an brauchbaren und durch Studien belegten Informationen in diesem Fachgebiet hin und beklagt das Fehlen zuverlässig durchgeführten Stu- 
dien, die den Kriterien der evidenzbasierten Medizin entsprechen. So ist die Anregung solcher uni- und multizentrischen Studien sicherlich ein wertvoller Nebeneffekt dieses Buchs. $\mathrm{Zu}$ seinem Erfolg werden sicherlich die zahlreichen ausgezeichneten Farbphotos sowie die Darstellung auch interventioneller Verfahren mit der Demonstration von Eingriffen durch Bildsequenzen beitragen. Stets stellt der Autor sein Vorgehen in Frage und ist auf der Suche nach Verbesserungsmöglichkeiten - eine Strategie, die gerade vor dem Hintergrund der unsicheren Datenlage Schule machen sollte. Letztendlich ist sein Preis schon in Anbetracht des umfangreichen Bildmaterials sehr günstig.

Karl-Joseph Paquet, Bad Kissingen

\section{Reanimation - Empfehlungen für die Wiederbelebung}

Bundesärztekammer (Hrsg)

Köln, Deutscher Ärzte-Verlag, 2007, 4., überarb. Aufl., XIX + 159 S., 42 Abb., 29,95 EUR

ISBN 978-3-7691-0529-2

Dieses wichtige Büchlein wurde 1991 erstmalig veröffentlicht und von Ärzten der unterschiedlichen Fachgebiete unter Beteilung der Bundesarbeitsgemeinschaft Erste Hilfe (BAGEH) erstellt. Es vermittelt die Grundlage für ein einheitliches Handeln beim Versagen der Herz-Lungen-Funktion, die sogenannte kardiopulmonale Wiederbelebung, und erscheint bereits in der 4., natürlich dem neuesten Wissenstand angepassten, gründlich überarbeiteten Neuauflage. Gleichzeitig wurde dabei der internationale wissenschaftliche Konsensus berücksichtigt, wie z.B. die Empfehlungen des ILCOR (International Liaison on Resuscitation) sowie die aktuellen Leitlinien des ERC (European Resucsitation Council). Dieses Gesamtwissen ist die maßgebliche Arbeitsgrundlage für alle Ärzte, die notfallmedizinisch tätig sind, und natürlich auch für Bildungsinstitutionen und das im Rettungsfach tätige medizinische Personal. Besonderer Wert wurde dabei auf den Grundsatz: «einfach reanimieren» gelegt. Im Detail werden Basismaßnahmen, erweiterte Maßnahmen wie EKG-Diagnostik, Defibrillation, Luftwegsmanagement und Beatmung sowie Medikamenteneinnahme, die Notfalltherapie vital bedrohlicher Erkrankungen wie Myokardinfarkt, akutes Koronarsyndrom und Arrhythmien (in diesem Kapitel werden sogar aktuelle Studienergebnisse berücksichtigt), die Wiederbelebung von Kindern und Säuglingen und schließlich Sonderfälle (z.B. Schwangerschaft, Hypothermie (Unterkühlung) und Stromschlag) besprochen. Auch ethische Aspekte, Maßnahmen nach erfolgreicher Postreanimationsphase und einige Sonderfälle wie z.B. das Beinahe-Ertrinken werden erörtert. Das Buch ist das umfassende, deutsche Standardwerk auf dem Gebiet der Reanimation und wird daher ohne Ein- schränkungen nahezu allen Ärzten und dem ärztlichen Hilfspersonal, die fast täglich oder auch nur gelegentlich in der Herz-Lungen-Wiederbelebung engagiert sind bzw. sich engagieren sollten, ohne Einschränkung empfohlen. Sein Preis ist mehr als günstig.

Karl-Joseph Paquet, Bad Kissingen

\section{Gehirn}

K. Sartor, S. Hähnel, B. Kress (Hrsg.)

Stuttgart, Thieme, 2006, Pareto-Reihe Radiologie XI + 299 S., 336 Abb., 49,95 EUR

ISBN 978-3-13-137111-9

Die Pareto-Reihe Radiologie bietet nach dem Prinzip, dass 20\% der Diagnosen $80 \%$ des Arbeitsalltags ausmachen, einen guten Überblick über die jeweilige Untersuchungsregion. Der Band «Gehirn» ist in 13 Unterkapitel unterteilt. So werden z.B. im Kapitel «Trauma» typische Verletzungen wie das epidurale Hämatom und im Kapitel «Entzündungen» typische Erkrankungen wie die multiple Sklerose erläutert. Weitere Kapitel beschäftigen sich mit Hirnaneurysmen, Schlaganfällen, Erkrankungen der Hirnhäute sowie Erkrankungen der Ventrikel und Zisternen. Im Kapitel «Vaskuläre Erkrankungen» geht es um typische Erkrankungen wie die venöse Dysplasie, im Kapitel «Tumoren» um typische Befunde wie die Hirnmetastase, im Kapitel «Zysten» um bei Hirnzysten typische Befunde wie die Pinealiszyste, im Kapitel «Leukenzephalopathien» um typische Erkrankungen wie Morbus Alzheimer und im Kapitel «Kongenitale Fehlbildungen» um typische Erkrankungen wie die Chiari-Malformation. In den letzten beiden Kapiteln werden abschließend Artefakte in der MRT und postoperative Veränderungen besprochen. Jede der im Buch vorgestellten Erkrankungen wird nach dem gleichen Schema abgehandelt. Zuerst wird die Krankheit an sich mit Epidemiologie, Ätiologie, Pathophysiologie und Pathogenese erklärt. Anschließend werden die verschiedenen bildgebenden Verfahren, mit denen die Erkrankung untersucht werden kann, mit typischen Untersuchungsbefunden vorgestellt. Vor allem wird aber am Anfang die Methode der Wahl zur Untersuchung genannt und erläutert, was gerade für weniger erfahrene Radiologen in der Weiterbildung eine große Hilfe ist. Anschließend werden typische klinische Befunde und Behandlungsoptionen erklärt. Abgerundet wird die jeweilige Darstellung durch eine differentialdiagnostische Tabelle, weiterführende Literaturangaben und typisches Bildmaterial. Insgesamt sind die jeweiligen Besprechungen der Krankheitsbilder sehr gut gelungen, so etwa die sehr gelungene Beschreibung der Untersuchungsbefunde oder die hilfreichen Angaben zur weiteren Therapie, die beim beratenden Patientengespräch weiterhelfen. Teilweise gibt es jedoch für die 
nächste Auflage Verbesserungsbedarf. So sind nicht alle Abbildungen mit Pfeilen beschriftet, um die pathologischen Strukturen zu erkennen. Dies sollte geändert werden, so dass auf allen Abbildungen Pfeile vorhanden sind. Auch eine ergänzende CD-ROM mit den Bildern und einer Lupenfunktion wäre toll, da teilweise durch das kleine Format gerade auf den Röntgenbildern die Pathologien schwer zu erkennen sind. Auch sind teilweise nur Bilder einer Modalität (Röntgen, CT, MRT) abgebildet. Dies sollte insofern geändert werden, als dass zumindest von jeder empfohlenen Untersuchungsmethode charakteristische Bilder vorhanden sind. Dennoch kann ich das Buch uneingeschränkt jedem empfehlen, der sich mit der Bildgebung des Gehirns beschäftigen möchte. Der Preis ist angemessen.

Nicolas Paquet, Hannover

\section{Kompendium evidenzbasierte Medizin. Clinical Evidence Concise}

\section{G. Ollenschläger, H.C. Bucher, N. Donner-Banzhoff,}

J. Forster, W. Gaebel, R. Kunz, O.-A. Müller, E. Neugebauer,

J. Steurer (Hrsg.)

Bern, Hans Huber, 2007, 6. Aufl., XXXVIII + 1319 S.,

4 Abb., 43 Tab., 59,95 EUR

ISBN 978-3-456-84421-3

Medizinische Entscheidungen sind heute «evidence-based». Das Kompendium evidenzbasierte Medizin stellt zu den praktisch wichtigsten Krankheitsbildern die zentralen klinischen Fragen und dokumentiert davon ausgehend verlässliche Ergebnisse zu einzelnen Therapieoptionen. Das Werk ist in großen Teilen die Übersetzung eines internationalen, vom Verlag der British Medical Association herausgegebenen Buchprojekts. Jährlich überarbeitet und neu aufgelegt, haben an der aktuellen Auflage mehr als 100 Experten mitgearbeitet, die Tausende von Studien zusammengetragen und auf ihre Praxisrelevanz überprüft haben. Obwohl das Spektrum der abgehandelten Themen sich über die gesamte Medizin erstreckt, sind leider immer noch viele Krankheitsbilder nicht erwähnt. So werden im Kapitel hämatologische Erkrankungen nur das multiple Myelom, das Non-Hodgkin-Lymphom und die Sichelzellkrankheit kommentiert. Auch sind Suchdaten $\mathrm{zu}$ den einzelnen Krankheitsbildern vorwiegend aus den Jahren 2004 und 2005, so dass hochaktuelle Erkenntnisse dazu fehlen bzw. nicht bewertet werden können. Trotz dieser Defizite, die nur als Beispiele aufgeführt sind und leider nicht die einzigen sind, kann dieses Kompendium den Arzt in der Praxis und im Krankenhaus bei zahlreichen, vor allem den häufigeren, Krankheitsbildern schnell, objektiv und meist aktuell informieren. Trotz des laufend aktualisierten und sicherlich sehr vorteilhaften Internet-Zugangs kann er längere Recherchen in diesem Medium vermeiden. Dies trifft auch auf den Gesellschaftsarzt der privaten und den Mitarbeiter des Medizinischen Dienstes der gesetzlichen Krankenversicherungen $\mathrm{zu}$; ihnen ist dieses Werk eine wertvolle Hilfe bei der Beurteilung der medizinischen Notwendigkeit umfangreicher und kostspieliger Behandlungen bzw. Operationen. Somit bietet das Kompendium aktuelle Zusammenfassungen der besten weltweit verfügbaren Belege $\mathrm{zu}$ den wichtigsten klinischen Fragestellungen und Behandlungsalternativen, klinische Informationen, von mehr als 100 ausgewiesenen deutschsprachigen Experten aus Tausenden von Studien zusammengetragen und auf ihre Praxisrelevanz geprüft sowie eine unverzichtbare Verdichtung neuester Forschungsergebnisse für den praktisch tätigen Arzt und bleibt dabei ohne Verzerrung durch subjektive Meinungen oder Interessen. Somit ist eigentlich der Besitz dieses Kompendiums für jeden Arzt, der sich nicht dem Vorwurf aussetzen will, sich nicht entsprechend dem hippokratischen Eid ständig fortzubilden bzw. zu informieren, trotz der oben aufgeführten Mängel unverzichtbar. Sein Preis ist günstig, dies gilt besonders für den Bezug im Abonnement.

Karl-Joseph Paquet, Bad Kissingen

\section{Was gibt es Neues in der Chirurgie?}

\section{K. Meßmer, J. Jähne, P. Neuhaus}

Landsberg-Lech, ecomed Medizin, 2007, 558 S., 159,-- EUR ISBN 978-3-609-76965-3

Dieses Buch hat sich schon seit Jahren einen festen und gesicherten Platz in der Fort- und Weiterbildungsliteratur für Chirurgen erobern können. Ziel der Buchreihe «Was gibt es Neues in der Chirurgie?» ist, die neuesten Entwicklungen und Standards in den einzelnen Gebieten der Chirurgie einschließlich aller Teilgebiete, zu denen auch die Kinder- und Handchirurgie gerechnet werden, präzise und klar strukturiert aufzuzeigen. Renommierte Chirurgen verfolgen fortlaufend die Entwicklungen in ihrem Spezialgebiet, sichten neue und neueste Literatur, werten Kongressberichte aus, spüren Trends auf und beschreiben anschaulich neueste Verfahren. Nur der Chirurg kann seine Patienten kompetent beraten und behandeln, der diese Entwicklungen kennt und auch interdisziplinäre Therapievorschläge erörtern kann. In diesem Jahresband spiegeln einige Beiträge wider, dass sich in der Chirurgie ein gravierender Wandel vollzieht. Besonders deutlich wird dies bei der molekularen Onkologie (siehe Beitrag von H. Allgayer, Mannheim, S. 29-53), gilt aber auch weniger ausgeprägt für andere Gebiete wie z.B. die Hightech-Chirurgie, die postoperative Schmerztherapie, das Qualitätsmanagement, die präoperative Risikoabschät- 
zung und die Fast-Track-Rehabilitation. Erstmals werden die Themen «Was gibt es Neues bei den Lebertumoren bzw. den Lebermetastasen?» separat dargestellt. Die Identifizierung molekulargenetischer Markermodule erlaubt erstmals, beim individuellen Tumorpatienten Voraussagen über dessen Ansprechen auf adjuvante und neoadjuvante Therapieoptionen $\mathrm{zu}$ machen. Beibehalten wurde dabei die Klassifizierung der zitierten Arbeiten nach den Prinzipien der Evidence Based Medicine (EBM) entsprechend den Richtlinien der AHCPR (US Agency for Health Care Policy and Research). Mit Hilfe eines Fragebogens kann sich jeder Nutzer bei der zuständigen Landesärztekammer zahlreiche Fortbildungspunkte für das Jahr 2007 zertifizieren lassen. Der Inhalt des Werks ist originell und kenntnisreich ausgesucht; seine Lektüre garantiert dem Chirurgen einen wesentlichen Fortschritt in seiner Weiterbildung. Die einzelnen Beiträge sind gut illustriert und mit einem aktuellen und umfangreichen Literatur- und Sachverzeichnis. Das Werk kann daher ohne wesentliche Einschränkung empfohlen werden. Sein Preis ist angemessen.

Karl-Joseph Paquet, Bad Kissingen

\section{Gefäßchirurgie}

W. Hepp, H. Kogel (Hrsg.)

München, Urban Fischer bei Elsevier, 2007, 2., aktualis. u. erw. Aufl., XII + 819 S., 260 Abb., davon 178 farb., 71 Tab., 229,- EUR

ISBN 978-3-437-21841-5

Das vorliegende Buch hat sich zum Ziel gesetzt, den angehenden Gefäßchirurgen prägnant und schnell in die schwierige Materie der modernen Gefäßchirurgie des 21. Jahrhunderts einzuführen. Dies gilt ebenso für den Allgemeinchirurgen mit gefäßchirurgischem Interesse wie auch für Ärzte aus kooperierenden Disziplinen (Angiologie, interventionelle Radiologie, Kardiochirurgie), denen durch dieses Buch auch die Denkweise des Gefäßchirurgen nahegebracht werden soll. Aufbauend auf ihre eigenen jahrzehntelangen klinischen Erfahrungen stellen die Autoren alle relevanten Fakten didaktisch überzeugend, knapp und mit zahlreichen anschaulichen Abbildungen dar. Das Werk steht gewissermaßen wie ein Kochbuch zwischen Operationslehre und wissenschaftlichem Einstieg. Es beginnt mit einem allgemeinen Teil, in dem alle grundlegenden Aspekte der Anatomie, Physiologie und Pathophysiologie, Diagnostik und Dokumentation sowie die Grundzüge der Begutachtung erörtert werden. Der spezielle Teil beschreibt Angiodysplasien, Gefäßverletzungen, Kompressionssyndrome, Aneursymen, akute arterielle Verschlüsse, chronische arterielle Verschlussprozesse, extraanatomische Verfahren, gefäßchirurgische Zugangseingriffe, nicht- rekonstruktive flankierende Eingriffe und die vaskuläre Tumorchirurgie. Das epifasziale und tiefe Venensystem unter Einbeziehung von akuten Verschlussprozessen und chronischen Verschlüssen, das Lymphgefäßsystem sowie postoperative Komplikationen in der Gefäßchirurgie kommen ebenfalls zur Sprache. In der Neuauflage wurden im Hinblick auf die rasante Entwicklung der Gefäßchirurgie besonders die Kombinationseingriffe und Hybridprozeduren berücksichtigt, die auf indikatorischen und operativen Neuerungen vor allem durch Fortschritte der endovaskulären Methoden beruhen. Erstmals eingearbeitet wurden die Leitlinien der Deutschen Gesellschaft für Gefäßchirurgie. Somit deckt das Buch das neue Curriculum der Weiterbildung in der Gefäßchirurgie komplett ab. Die gewählte Darstellung der Thematik ist wie in der Erstauflage inhaltlich umfassend, didaktisch geschickt und mit zahlreichen dreifarbigen Tabellen und Abbildungen sowie weiteren Schwarz-Weiss-Abbildungen gut illustriert. Die Literaturverzeichnisse enthalten die wesentlichen Zitate und ermöglichen es dem engagierten Leser, noch tiefer in die Materie einzudringen. Das Sachverzeichnis ist mit 40 Seiten sehr umfangreich. Somit kann diese Neuauflage nur als sehr gelungen bezeichnet werden, weshalb ihr eine weite Verbreitung im angesprochenen Leserkreis gewünscht wird. Der Preis ist angemessen.

Karl-Joseph Paquet, Bad Kissingen

\section{Pschyrembel Sozialmedizin}

Berlin, Walter de Gruyter, 2007, 552 S., 39,95 EUR ISBN 978-3-11-017605-6

Neu erschienen in der Pschyrembel-Lexikon-Reihe ist Pschyrembel Sozialmedizin mit den Untertiteln Gesundheitssystem, Public Health, Sozialrecht, Rehabilitation, Prävention und Gesundheitsökonomie. Dieses Werk entstand aufgrund einer Idee in der Abteilung Sozialmedizin der ehemaligen Bundesversicherungsanstalt für Angestellte (heute Deutsche Rentenversicherung Bund). Daher stammen die Bearbeiter (eigentlich Herausgeber) aus dem Geschäftsbereich Sozialmedizin und Rehabilitationswissenschaft der Deutschen Rentenversicherung Bund. Um den umfassenden Anspruch der Sozialmedizin zu erfüllen, werden in diesem Lexikon die über das System der sozialen Sicherung hinausgehenden Themengebiete der Epidemiologie, der Statistik, der Gesundheitsund Sozialökonomie, des heute besonders wichtigen Qualitätsmanagements, der Psychologie, der Umweltmedizin, der Forschung und Lehre sowie der sozialmedizinisch relevanten Berufbilder aufgenommen. Das Werk wurde primär für Ärzte in Rehabilitations- und Akutkliniken, in der Sozialversicherung und in der niedergelassenen Praxis konzipiert. Diesen großen ärztlichen Berufsgruppen bietet es mit seinem multi- 
dimensionalen Ansatz wichtige und umfassende Informationen zu den oben aufgeführten Themen. Wie breit diese dargestellt werden, zeigt der immer wieder deutliche interdisziplinäre Ansatz des Autorenteams, dem neben Ärzten und Juristen auch Psychologen, Ökonomen und Gesundheitswissenschaftler angehören. Das vorgelegte Wissen wird, entsprechend den bekannten Qualitätsanforderungen der bisherigen Pschyrembel-Familie und unter den Aspekten Konsensfähigkeit, wissenschaftlicher Belegbarkeit und Aktualität, didaktisch ansprechend und qualitätsvoll präsentiert. Obwohl der Pschyrembel Sozialmedizin ein Erstlingswerk ist und die Aufgabe seiner Erstellung von den Herausgebern anfänglich unterschätzt worden ist, ist ihnen ein großer Wurf gelungen, der dem oben angesprochenen Personenkreis ohne Einschränkung empfohlen werden kann.

Karl-Joseph Paquet, Bad Kissingen

\section{Risikomanagement in der operativen Medizin}

\author{
J. Enneker, D. Pietrowski, P. Kleine \\ Darmstadt, Steinkopff, 2007, VIII + 189 S., 33 Abb., \\ 49,95 EUR
}

ISBN 978-3-7985-1737-0

Niemand macht gerne Fehler. Dennoch ereignen sie sich täglich. Besonders unangenehm und folgenreich sind Behandlungsfehler in der Medizin. Sie können sich leicht zur Katastrophe entwickeln. Erst danach sucht der Mediziner (meist der Chirurg) nach Fehlerursachen, die zu diesem Unglück geführt haben. Danach wird häufig ein Verursacher gefunden und beschuldigt. Damit meinen die meisten Mediziner, ihrer Verantwortung genügt zu haben. Inzwischen weiß man allerdings, dass solche Fehler selten auf eine Person zurückzuführen sind, sondern häufig äußere Ursachen für die Fehlerentstehung verantwortlich sind. Somit wäre es viel besser, vorher die Ursachen zu kennen oder nach ihnen zu suchen und damit einen größeren Schaden zu vermeiden. Dass dies möglich ist, zeigen Untersuchungen aus der Hochrisikoindustrie wie z.B. der Luftfahrt oder Kernkraft. Die Herausgeber - verstärkt durch hochqualifizierte Autoren aus nichtmedizinischen Bereichen wie Juristen und Versicherungswissenschaftler haben im vorliegenden Werk dargestellt, wie Risikovermeidung und -management in der Medizin aussehen sollte. Jedes Kapitel beginnt mit einem humorvollen Leitsatz. Die Kapitel sind meist durch eine oder mehrere Abbildung(en) bzw. Tabellen illustriert und haben häufig ein Literaturverzeichnis, oder dieses findet sich am Buchende. Ergänzt werden alle Spezialkapitel durch eine Zusammenfassung der Berichtssysteme aus anderen Ländern, ein Glossar und ein aussagekräftiges Sachverzeichnis. Dieses Buch füllt sicherlich ein Lücke und wird hoffentlich dazu beitragen, in Zukunft Behand- lungsfehler zu vermeiden und/oder sie nach ihren unbeabsichtigten Auftreten richtig zu managen. Somit sollte jeder Mediziner, insbesondere aber jeder operativ Tätige, dieses Buch lesen. Sein Preis ist angemessen.

Karl-Joseph Paquet, Bad Kissingen

\section{Arzneimittel, 2 Ordner zur Fortsetzung. Ein Handbuch für Ärzte und Apotheker}

\section{H. Hellwig, H.-H. Otto}

Stuttgart, Wissenschaftliche Verlagsgesellschaft mbH, 2007, Losebl.-Ausg., 10. Aufl. Stand 6. Erg.-Lfg, 270,- EUR ISBN 978-3-8047-2361-0

Der tatsächliche Nutzen neuer Arzneimittel ist bei ihrer Einführung trotz zahlreicher vorangegangener Studien nicht bekannt oder sehr schwer zu beurteilen. Erst durch den breiten Einsatz in Klinik und Praxis kristallisieren sich die Vorund Nachteile heraus. Diesem Problem können alle Therapeuten und Ärzte durch die sorgfältige Lektüre des Loseblattgroßwerkes «Arzneimittel» entgehen. Seine Autoren informieren aktuell über den sich häufig ändernden Arzneimittelmarkt und seine ganze Breite. Diese zweibändige, umfangreiche Loseblattsammlung, die ständig aktualisiert wird, hat sich seit mehr als 50 Jahren bei Apothekern und Ärzten in Forschung und Praxis bewährt und ist immer aktuell. Sie ist ähnlich wie die Rote Liste strukturiert, übersichtlich angeordnet und enthält für jedes Arzneimittel die chemische Formel. Alle Kapitel sind auf dem neuesten Kenntnisstand und bieten eine umfassende und kritische Darstellung der zahlreichen neuen Substanzen, basierend auf theoretischen und klinischen Fakten sowie bekannt gewordenen und unerwünschten Wechselwirkungen. Besonders umfangreiche Neuerungen sind bei Immuntherapeutika, Magen-Darm-Mitteln, Psychopharmaka, Diagnostika und Tumortherapeutika zu finden. Darüber hinaus werden nahezu alle Monopräparate und ausgewählte Kombinationen erfasst und umfassend, aber stets kritisch dargestellt. Die Neueinführungen werden im Vergleich mit der Standardtherapie bewertet. Die Aktualisierungslieferung berücksichtigt alle bis September 2006 in Verkehr gekommenen Analgetika, Blut beeinflussende Arzneimittel, Gynäkologika und Hormone. Seit mehr als drei Generationen informiert dieses Werk unabhängig, aktuell und kompetent. Trotz seines hohen, aber in Anbetracht seines extremen Umfangs und seiner ständigen Aktualisierung angemessenen Preises wird dieses Standardwerk für Ärzte und Apotheker ohne Einschränkung empfohlen und muss als unverzichtbar für diese Berufsgruppen bezeichnet werden.

Karl-Joseph Paquet, Bad Kissingen 


\section{Atlas der laparokopischen Chirurgie. Operationsablauf,} Varianten, Komplikationen

\section{T. Carus}

Berlin, Springer, 2007, XII + 334 S., 644 Farbabb., 129,95 EUR ISBN 978-3-540-33673-0

Die laparoskopische Chirurgie, die mit der Cholezystektomie begann, hat eine stürmische Entwicklung genommen, die keineswegs abgeschlossen ist. Die laparoskopische Cholezystektomie ist inzwischen Gold-Standard. Dazu haben nicht zuletzt die Patienten beigetragen, die wegen reduzierter Schmerzen, schnellerer Rekonvaleszenz und besserer Kosmetik diese Verfahren von Beginn an favorisierten. Ständig werden neue Anwendungen und Techniken in großer Zahl und rascher Folge entwickelt, die nicht nur resezierende, sondern auch rekonstruktive laparoskopische Operationen ermöglichen. Inzwischen ist außer der Cholezystektomie, Appendektomie und Herniotomie auch das laparoskopische Vorgehen bei der Fundoplicatio, der Darmresektion und in der Adipositas- und Milzchirurgie anerkannt. Vereinzelt werden sogar laparoskopische Operationen am Pankreas erfolgreich durchgeführt. Zwar gibt es viele Bücher und vor allem Kongressberichte über die laparoskopische Chirurgie, und ihre Darstellung hat Eingang in die chirurgische Operationslehre gefunden. Dennoch fehlte ein Standardwerk bzw. ein Atlas zu diesen Verfahren; dies(en) hat der erfahrene laparoskopische Chirurg Thomas Carus jetzt vorgelegt. Es ist in einen allgemeinen Teil, nämlich die Darstellung der Grundlagen (Operationsindikation und -kontraindikation, Instrumente, Operationsvorbereitung und Nahttechniken) und in einen speziellen Teil gegliedert. Im Letzteren werden in ausführlichen Kapiteln die diagnostische Laparoskopie, die laparokopische Adhäsiolyse und Bridendurchtrennung, die Probeexzision und Biopsie, die Therapie von Perforationen und Eingriffe am Zwerchfell und Magen, der Leber, der Gallenblase und den Gallengängen, am Dünndarm, Kolon und Rektum sowie bei Hernien beschrieben. Diese Beschreibung erfolgt detailliert, didaktisch geschickt und hervorragend illustriert. Immerhin finden sich weit über 600 farbige Abbildungen auf 334 Seiten, somit 1-7 Abbildungen pro Seite, ergänzt durch zweifarbige Tabellen. Die Abbildungen sind von einzigartiger Qualität und Aussagekraft. Jedes Detail beim laparoskopischen Vorgehen kann leicht nachvollzogen werden. Dass das Literaturverzeichnis sehr kurz geraten ist, wird überzeugend erklärt. Dafür ist das Sachverzeichnis aussagekräftig. Dieses Werk muss man allen Viszeralchirurgen, insbesondere wenn sie schwerpunktmäßig laparoskopisch tätig sind, ohne Einschränkung empfehlen. Voraussichtlich wird es sich zum Standardwerk auf diesem Gebiet entwickeln. In Anbetracht seines umfassenden Inhalts und der großzügigen farbigen Gestaltung ist sein Preis als günstig einzustufen.

Karl-Joseph Paquet, Bad Kissingen

\section{Wirbelsäule}

H. Imhof (Hrsg.)

Stuttgart, Thieme, 2006, Pareto-Reihe Radiologie X + 301 S., 327 Abb., 49,95 EUR

ISBN 978-3-13-137141-6

Die Pareto-Reihe Radiologie bietet nach dem Prinzip, dass 20\% der Diagnosen 80\% des Arbeitsalltags ausmachen, einen guten Überblick über die jeweilige Untersuchungsregion. Der Band «Wirbelsäule» ist in 8 Unterkapitel unterteilt. So werden z.B. im Kapitel «Fehlbildungen» typische Erkrankungen wie die Meningozele, im Kapitel «Trauma» typische Frakturen wie die Densfraktur, im Kapitel «Degenerative Erkrankungen» typische Befunde wie der Bandscheibenvorfall, im Kapitel «Entzündungen» typische Erkrankungen wie die rheumatoide Arthritis, im Kapitel «Tumoren» typische Befunde wie das Plasmazytom, im Kapitel «Vaskuläre Erkrankungen» typische Erkrankungen wie die AV-Malformation, im Kapitel «Postoperative Veränderungen» typische Befunde wie die Periduralfibrose und im Kapitel «Stoffwechselerkrankungen» typische Erkrankungen wie der Morbus Paget erläutert. Jede der im Buch vorgestellten Erkrankungen wird nach dem gleichen Schema abgehandelt. Zuerst wird die Krankheit an sich mit Epidemiologie, Ätiologie, Pathophysiologie und Pathogenese erklärt. Anschließend werden die verschiedenen bildgebenden Verfahren vorgestellt, mit denen die Erkrankung mit typischen Untersuchungsbefunden diagnostiziert werden kann. Vor allem wird aber am Anfang die Methode der Wahl zur Untersuchung genannt und erläutert, was gerade für weniger erfahrene Radiologen in der Weiterbildung eine große Hilfe ist. AnschlieBend werden typische klinische Befunde und Behandlungsoptionen erläutert. Abgerundet wird die jeweilige Darstellung durch eine differentialdiagnostische Tabelle, weiterführende Literaturangaben und typisches Bildmaterial. Insgesamt sind die jeweiligen Besprechungen der Krankheitsbilder sehr gut gelungen, so etwa die sehr gute Beschreibung der Untersuchungsbefunde oder die hilfreichen Angaben zur weiteren Therapie, die beim beratenden Patientengespräch weiterhelfen. Teilweise sind jedoch für die nächste Auflage Verbesserungsvorschläge zu machen. So sind nicht alle Abbildungen mit Pfeilen beschriftet, um die pathologischen Strukturen zu erkennen. Dies sollte geändert werden. Auch eine ergänzende CDROM mit den Bildern und einer Lupenfunktion wäre toll, da teilweise durch das kleine Format gerade auf den Röntgenbildern die Pathologien schwer zu erkennen sind. Auch sind teilweise nur Bilder einer Modalität (Röntgen, CT, MRT) abgebildet. Dies sollte insofern verbessert werden, dass zumindest von jeder empfohlenen Untersuchungsmethode charakteristische Bilder vorhanden sind. Trotzdem kann ich das Buch uneingeschränkt jedem empfehlen, der sich mit der Bildgebung der Wirbelsäule beschäftigen will. Der Preis ist angemessen.

Nicolas Paquet, Hannover 